Research Article

\title{
Integral Sliding Mode Control of Lur'e Singularly Perturbed Systems
}

\author{
Yanyan Wang ${ }^{1}$ and Wei Liu ${ }^{1,2}$ \\ ${ }^{1}$ School of Mathematics and Statistics, Zhoukou Normal University, Zhoukou 466001, China \\ ${ }^{2}$ Center for Applied and Interdisciplinary Mathematics, Department of Mathematics, East China Normal University, \\ Shanghai 200241, China
}

Correspondence should be addressed to Wei Liu; liuweiecnu@163.com

Received 1 April 2015; Revised 23 August 2015; Accepted 24 August 2015

Academic Editor: Antonios Tsourdos

Copyright (C) 2015 Y. Wang and W. Liu. This is an open access article distributed under the Creative Commons Attribution License, which permits unrestricted use, distribution, and reproduction in any medium, provided the original work is properly cited.

\begin{abstract}
This paper investigates the integral sliding mode control problem for Lur'e singularly perturbed systems with sector-constrained nonlinearities. First, we design a proper sliding manifold such that the motion of closed-loop systems with a state feedback controller along the manifold is absolutely stable. To achieve this, we give a matrix inequality-based absolute stability criterion; thus the above problem can be converted into a matrix inequality feasibility problem. In addition, the gain matrix can also be derived by solving the matrix inequality. Then, a discontinuous control law is synthesized to force the system state to reach the sliding manifold and stay there for all subsequent time. Finally, some numerical examples are given to illustrate the effectiveness of the proposed results.
\end{abstract}

\section{Introduction}

Singularly perturbed systems are commonly encountered due to the presence of small parasitic parameters such as small time constants and moments of inertia. These are often the source of the increased order and stiffness of systems [1,2], where robust stability is a crucial requirement for singularly perturbed systems. This is due to not only theoretical interests but also the relevance of this topic in control engineering applications. During the past years, the robust stability and stabilization problems of singularly perturbed systems have been widely studied, and many significant results and methods have been presented (see [3-16] and the references therein). Reference [3] presented a composite linear controller for robust stability of singularly perturbed linear systems with matching condition uncertainties, in which the maximum stability bound has not been involved. In [5], the robust stabilization problem of singularly perturbed systems with nonlinear uncertainties is studied; a control law is presented by the solutions of two independent Lyapunov equations. In addition, the stability bound is also derived via a state transformation and the constructive use of a Lyapunov function. The obtained result shows that the controller design approach is very complex and difficult to operate. More recently, the authors of [9] considered absolute stability problem for Lur'e singularly perturbed systems, where the stability criterion in terms of linear matrix inequalities (LMIs) is obtained. Meanwhile, the maximum stability bound is also computed by the bisectional search algorithm.

For robust control strategy, sliding mode control (SMC) provides an efficient way to control high-order nonlinear dynamic systems operating under uncertain conditions, which has many advantages like ease of implementation, fast response, and reduction in the order of the state equation. The basic idea of SMC is to drive the state trajectory of the system onto some specified smooth manifold (sliding surface) passing through the zero state in the state space and maintain the trajectory on it for all subsequent time. Recently, considerable attention has been paid to it and significant advances have been made on this regard [17-25]. Reference [23], for example, considered the sliding mode control for a class of uncertain singularly perturbed systems; a sufficient condition for the stability of control systems in terms of LMIs with equality constraint is presented. The obtained result is novel 
and interesting. The only downside is the fact that, owing to the equality constraint, the search of the controller gain is difficult and complex. However, to the best of authors' knowledge, SMC for Lur'e singularly perturbed systems has seldom been investigated so far, although it has many profound engineering applications. Since a direct application on SMC methods of normal systems to Lur'e singularly perturbed systems may lead to ill-conditioned issue, many difficult and efficient issues still need to be addressed.

Motivated by the above works, we, in this paper, consider integral SMC for Lur'e singularly perturbed systems, although the absolute stability of such a system without control input has been studied in [9]. Our concern is whether stabilization of the closed-loop system can be achieved when considering SMC. To this end, we first select a proper integral sliding manifold such that the sliding mode dynamics is absolutely stable in the specified switching surface, in which the sliding mode gain matrix can be obtained by solving a matrix inequality. In addition, equality constraint in solving the matrix inequality can also be avoided. Then, a discontinuous control law is designed to force the original system state to reach the sliding surface and stay there for all future time. Moreover, the maximum stability bound is also derived by using the bisectional search algorithm proposed in [9]. Finally, two illustrative examples are presented to show the effectiveness of the presented approach.

The rest of the paper is organized as follows. Section 2 gives the problem formulation. The main results are given in Section 3. Section 4 gives two examples to show the effectiveness of the proposed methods. Finally, the conclusion is drawn in Section 5.

\section{Problem Formulation}

Consider the following Lur'e singularly perturbed systems given by

$$
\begin{aligned}
E_{\varepsilon} \dot{x}(t) & =A x(t)+B \varphi(y)+B_{u} u(t), \\
y(t) & =C x(t),
\end{aligned}
$$

where $x(t)=\left(x_{1}^{T}(t), x_{2}^{T}(t)\right)^{T}$ is the system state, $x_{1} \in R^{n}$ is the slow state, and $x_{2} \in R^{m}$ is the fast state; $u \in R^{p}$ is the control input vector; $y \in R^{q}$ is the output vector, $A, B, B_{u}$, and $C$ are constant matrices with appropriate dimensions; the matrix $E_{\varepsilon}$ is given by

$$
E_{\varepsilon}=\left(\begin{array}{cc}
I_{n} & O \\
O & \varepsilon I_{m}
\end{array}\right)
$$

where $\varepsilon$ is the perturbation parameter which is small and positive but may be unknown, representing the response of the fast dynamics. $\varphi(y)$ is vector-value continuous nonlinear function, which is assumed to satisfy the following sector condition:

$$
\varphi^{T}(y)(\varphi(y)-L y) \leq 0, \quad y \in R^{q}
$$

or

$$
0 \leq \frac{\varphi_{i}\left(y_{i}\right)}{y_{i}} \leq l_{i}, \quad i=1,2, \ldots, q,
$$

where $L=\operatorname{diag}\left(l_{1}, l_{2}, \ldots, l_{q}\right)>0$ and $\varphi(y)=\left(\varphi_{1}\left(y_{1}\right) \varphi_{2}\left(y_{2}\right)\right.$ $\left.\cdots \varphi_{q}\left(y_{q}\right)\right)^{T}$.
Remark 1. The structure of the sector condition in the form of (4) or (5) has been widely considered (see $[6,7,26,27]$ and the references therein). As stated in [9], this sector structure does not involve any approximation of nonlinearities by their norms. Thus, it is expected that our method can lead to a reduced conservation in controller design and stability bound.

We now give some basic results before continuing our discussion, which will be useful for the stability analysis of the sliding mode dynamics.

Lemma 2 (see [28]). Assume that $H, D$, and $E$ are real matrices with appropriate dimensions and $F(t)$ is a real matrix function satisfying $F^{T}(t) F(t) \leq I$. Then

(1) $H+D F(t) E+(D F(t) E)^{T}<0$ holds if and only if there exists a scalar $\varepsilon>0$ satisfying $H+\varepsilon D D^{T}+\varepsilon^{-1} E^{T} E<0$;

(2) For any $\varepsilon>0$, we have $D F(t) E+(D F(t) E)^{T} \leq \varepsilon D D^{T}+$ $\varepsilon^{-1} E^{T} E<0$.

Lemma 3 (see [9]). If there exists a diagonal matrix $\Gamma>0, a$ lower triangular matrix

$$
P=\left(\begin{array}{ll}
P_{11} & O \\
P_{21} & P_{22}
\end{array}\right)
$$

with $0<P_{11} \in R^{n \times n}$ and $0<P_{22} \in R^{m \times m}$, and $\Lambda=$ $\operatorname{diag}\left\{\begin{array}{llll}\lambda_{1} & \lambda_{2} & \cdots & \lambda_{q}\end{array}\right\}$ with $\lambda_{i}=0$ when $c_{1}^{i} \neq 0$ and $c_{2}^{i} \neq 0$, satisfying the following linear matrix inequality:

$$
\begin{aligned}
\Phi & =\left(\begin{array}{cc}
A^{T} P+P^{T} A & P^{T} B+A^{T} C^{T} \Lambda+C^{T} L \Gamma \\
B^{T} P+\Lambda C A+\Gamma L C & \Lambda C B+B^{T} C^{T} \Lambda-2 \Gamma
\end{array}\right) \\
& <0,
\end{aligned}
$$

where $C=\left(\begin{array}{lll}C_{1} & C_{2}\end{array}\right)=\left(\begin{array}{llll}c_{1} & c_{2} & \cdots & c_{q}\end{array}\right)^{T}$ and $c_{i}=\left(\begin{array}{ll}c_{i}^{1} & c_{i}^{2}\end{array}\right.$ $\left.\cdots \quad c_{i}^{q}\right)^{T}$. Then there exists $\varepsilon_{1}^{*}>0$ such that system (1)-(2) with $u=0$ is absolutely stable for $\varepsilon \in\left(0, \varepsilon_{1}^{*}\right]$.

\section{Main Results}

In this section, we will first construct a proper integral sliding manifold such that the motion of system (1)-(2) along the manifold is absolutely stable; then, we design an integral SMC law such that the state of systems can be driven toward the manifold and stay there for all subsequent time regardless of the sector-constrained condition.

In the integral SMC approach, a law of the form

$$
u(t)=u_{0}(t)+u_{1}(t)
$$

is proposed, where $u_{0}(t)$ is the ideal control responsible for the performance of the nominal system; $u_{1}(t)$ is a discontinuous control providing the complete compensation of nonlinear uncertainties and ensuring that control objectives are reached. The sliding manifold is defined by the set $\{x \mid$ $s(x)=0\}$, with

$$
s(x(t))=G\left(E_{\varepsilon} x(t)-\int_{0}^{t}\left(A x(\tau)+B_{u} u_{0}(\tau)\right) d \tau\right),
$$

where $G \in R^{p \times(n+m)}$ is a projection matrix, satisfying the following. 
Assumption 4. The matrix product $G B_{u}$ is invertible.

Remark 5. The term

$$
\int_{0}^{t}\left(A x(\tau)+B_{u} u_{0}(\tau)\right) d \tau
$$

in (9) can be thought as a trajectory of the system in the absence of sector conditions and in the presence of the control $u_{0}(t)$. With this in mind, $s(x(t))$ can be considered a penalizing factor of the difference between the actual and the nominal trajectories, projected along $G$. Moreover, notice that the integral sliding surface constructed in (9) is different from that of [20], since the matrix $E_{\varepsilon}$ is taken into account; this enables avoiding some difficulties caused by the small parameter $\varepsilon$ in deriving the sliding mode dynamics in the sequel.

In this paper, we restrict ourselves to control functions in the form of static linear feedback

$$
u_{0}(t)=K x(t)
$$

where $K \in R^{p \times(n+m)}$ is a controller gain matrix.

To determine the motion equation at the sliding manifold, we use the equivalent control method [18]. According to (8) and (11), the solution of $E_{\varepsilon} x(t)$ is given by

$$
\begin{aligned}
& E_{\varepsilon} x(t)=E_{\varepsilon} x(0)+\int_{0}^{t}(A x(\tau)+B \varphi(y(\tau)) \\
& \left.+B_{u}\left(K x(\tau)+u_{1}(\tau)\right)\right) d \tau .
\end{aligned}
$$

It follows from (9) and (11) that

$$
\begin{gathered}
s(x(t))=G\left(E_{\varepsilon} x(0)+\int_{0}^{t}(A x(\tau)+B \varphi(y(\tau))\right. \\
\left.+B_{u}\left(K x(\tau)+u_{1}(\tau)\right)\right) d \tau-\int_{0}^{t}(A x(\tau)
\end{gathered}
$$

$$
\begin{aligned}
& \left.\left.+B_{u} u_{0}(\tau)\right) d \tau\right)=G\left(E_{\varepsilon} x(0)+\int_{0}^{t}(B \varphi(y(\tau))\right. \\
& \left.\left.+B_{u} u_{1}(\tau)\right) d \tau\right) .
\end{aligned}
$$

The derivative of $s(x(t))$ along time is

$$
\dot{s}(x(t))=G\left(B \varphi(y(t))+B_{u} u_{1}(t)\right) ;
$$

the equivalent control law can be obtained by solving the equation $\dot{s}(x(t))=0$ for $u_{1}(t)$;

$$
u_{1 \mathrm{eq}}=-\left(G B_{u}\right)^{-1} G B \varphi(y(t)) .
$$

By substituting $u_{1 \text { eq }}$ for $u_{1}(t)$ in (1), we obtain the following sliding dynamics:

$$
\begin{aligned}
E_{\varepsilon} \dot{x}_{\mathrm{eq}}(t) & =\bar{A} x_{\mathrm{eq}}(t)+\bar{B} \varphi(y), \\
y(t) & =C x_{\mathrm{eq}}(t),
\end{aligned}
$$

where $\bar{A}=\left(A+B_{u} K\right)$ and $\bar{B}=B-B_{u}\left(G B_{u}\right)^{-1} G B$.

The following result presents a sufficient condition via linear matrix inequalities technique which guarantees the absolute stability of the sliding mode dynamics (16).

Theorem 6. If there exists a scalar $\eta>0$, a diagonal matrix $\Gamma>0$, a matrix $\Lambda=\operatorname{diag}\left\{\begin{array}{llll}\lambda_{1} & \lambda_{2} & \cdots & \lambda_{q}\end{array}\right\}$ with $\lambda_{i}=0$ when $c_{1}^{i} \neq 0$ and $c_{2}^{i} \neq 0$, and a lower triangular matrix

$$
X=\left(\begin{array}{cc}
X_{11} & 0 \\
X_{21} & X_{22}
\end{array}\right)
$$

with $0<X_{11} \in R^{n \times n}$ and $0<X_{22} \in R^{m \times m}$, satisfying the following matrix inequality:

$$
\Phi_{1}=\left(\begin{array}{cccc}
A^{T} X+X^{T} A & A^{T} C^{T} \Lambda+C^{T} L \Gamma+X^{T} \bar{B} & \eta K^{T} B_{u}^{T} & X^{T} \\
\bar{B}^{T} X+\Lambda C A+\Gamma L C & \Lambda C \bar{B}+\bar{B}^{T} C^{T} \Lambda-2 \Gamma & O & \Lambda C \\
\eta B_{u} K & O & -\eta I & O \\
X & C^{T} \Lambda & O & -\eta I
\end{array}\right)<0,
$$

and then there exists an $\varepsilon^{*}>0$ such that system (16) is absolutely stable for $\varepsilon \in\left(0, \varepsilon^{*}\right]$.
Proof. By Schur's Complement Lemma, we can obtain that inequality (18) is equivalent to

$$
\left(\begin{array}{cc}
A^{T} X+X^{T} A+\eta K^{T} B_{u}^{T} B_{u} K+\eta^{-1} X^{T} X & A^{T} C^{T} \Lambda+C^{T} L \Gamma+X^{T} \bar{B}+\eta^{-1} X^{T} C^{T} \Lambda \\
\bar{B}^{T} X+\Lambda C A+\Gamma L C+\eta^{-1} \Lambda C X & \Lambda C \bar{B}+\bar{B}^{T} C^{T} \Lambda-2 \Gamma+\eta^{-1} \Lambda C C^{T} \Lambda
\end{array}\right)<0,
$$


which can be rewritten as

$$
\begin{gathered}
\left(\begin{array}{cc}
A^{T} X+X^{T} A & A^{T} C^{T} \Lambda+C^{T} L \Gamma+X^{T} \bar{B} \\
\bar{B}^{T} X+\Lambda C A+\Gamma L C & \Lambda C \bar{B}+\bar{B}^{T} C^{T} \Lambda-2 \Gamma
\end{array}\right) \\
+\eta\left(\begin{array}{c}
\left(B_{u} K\right)^{T} \\
O
\end{array}\right)\left(\begin{array}{ll}
B_{u} K & O
\end{array}\right) \\
+\eta^{-1}\left(\begin{array}{c}
X^{T} \\
\Lambda C
\end{array}\right)\left(\begin{array}{ll}
X & \left.C^{T} \Lambda\right)<0 .
\end{array}\right.
\end{gathered}
$$

$$
\bar{\Omega}=\left(\begin{array}{cc}
\left(A+B_{u} K\right)^{T} X+X^{T}\left(A+B_{u} K\right) & X^{T} \bar{B}+\left(A+B_{u} K\right)^{T} C^{T} \Lambda+C^{T} L \Gamma \\
\bar{B}^{T} X+\Lambda C\left(A+B_{u} K\right)+\Gamma L C & \Lambda C \bar{B}+\bar{B}^{T} C^{T} \Lambda-2 \Gamma
\end{array}\right)<0 .
$$

By applying Lemma 3, we obtain that there exists an $\varepsilon^{*}>0$ such that the sliding mode dynamics (16) is absolutely stable for $\varepsilon \in\left(0, \varepsilon^{*}\right]$. This completes the proof.

Remark 7. The above Theorem gives the first step of the SMC for Lur'e singularly perturbed system (1)-(2). Specifically, we design an integral-type switching surface as given in (9) so that the dynamics restricted to the switching surface (i.e., the sliding mode dynamics) has the form of (16). Observe that (18) is not a LMI due to the existence of the nonlinearity term $\eta B_{u} K$. Let $\bar{K}=\eta K$; then (18) can be transformed to a standard LMI which can be solved effectively by the LMI toolbox. Compared with [23], the derived sufficient condition for control law does not involve any equality constraint; its corresponding numerical problem can be avoided when using the LMI toolbox; this will help reduce any possibly induced conservatism.

Next, we will synthesize a proper SMC law to globally drive the system state trajectories onto the predefined switching surface $s(x(t))=0$ in finite time and maintain them there for all subsequent time. This is our second consideration of this paper.

In this paper, the discontinuous control $u_{1}(t)$ is selected as

$$
u_{1}(t)=-\rho(t) \frac{s(x(t))}{\|s(x(t))\|}
$$

where $\rho(t)$ is a gain high enough to enforce the sliding motion. We have the following result.

Theorem 8. Consider Lur'e singularly perturbed system (1)(2). Suppose that Assumption 4 holds and the matrix inequality (17)-(18) is solvable. Then, the trajectories of system (1)-(2) can be driven onto the switching surface $s(x(t))=0$ in a finite time by the following SMC law:

$$
u(t)=u_{0}(t)+u_{1}(t)
$$

According to Lemma 2, the above inequality is equivalent to

$$
\begin{aligned}
& \left(\begin{array}{cc}
A^{T} X+X^{T} A & A^{T} C^{T} \Lambda+C^{T} L \Gamma+X^{T} \bar{B} \\
\bar{B}^{T} X+\Lambda C A+\Gamma L C & \Lambda C \bar{B}+\bar{B}^{T} C^{T} \Lambda-2 \Gamma
\end{array}\right) \\
& \quad+\left(\begin{array}{c}
\left(B_{u} K\right)^{T} \\
O
\end{array}\right)\left(\begin{array}{ll}
X & C^{T} \Lambda
\end{array}\right)+\left(\begin{array}{c}
X^{T} \\
\Lambda C
\end{array}\right)\left(\begin{array}{ll}
B_{u} K & O
\end{array}\right) \\
& <0 ;
\end{aligned}
$$

thus we have

where $u_{0}(t)$ and $u_{1}(t)$ are defined by (11) and (23), respectively, with

$$
\rho(t)=\gamma+l\left\|\left(G B_{u}\right)^{-1} G B\right\|\|C x\|, \quad l=\max _{1 \leq i \leq q}\left\{l_{i}\right\} .
$$

Proof. To analyze the reachability of the specific switching surface, we choose $G$ as $G=B_{u}^{T} X$, where $X$ is a positive definite matrix. According to Assumption $4, G B_{u}=B_{u}^{T} X B_{u}$ is nonsingular and positive definite. Choose the following Lyapunov function:

$$
V(t)=\frac{1}{2} s^{T}(x(t))\left(B_{u}^{T} X B_{u}\right)^{-1} s(x(t)) .
$$

Following from (14) and (23), we have

$$
\dot{s}(x(t))=G\left(B \varphi(y(t))-B_{u} \rho(t) \frac{s(x(t))}{\|s(x(t))\|}\right) .
$$

Then, taking the derivative of $V(t)$ and considering the above equation, we have

$$
\begin{gathered}
\dot{V}(t)=s^{T}(x(t))\left(B_{u}^{T} X B_{u}\right)^{-1} \dot{s}(x(t))=s^{T}(x(t)) \\
\cdot\left(G B_{u}\right)^{-1} G\left(B \varphi(y(t))-B_{u} \rho(t) \frac{s(x(t))}{\|s(x(t))\|}\right) \\
\leq\|s(x(t))\|\left(l\left\|\left(G B_{u}\right)^{-1} G B\right\|\|C x(t)\|-\rho(t)\right) \\
\quad=-\gamma\|s(x(t))\| \leq-\alpha \sqrt{V(t)},
\end{gathered}
$$

where $\alpha=\sqrt{2 \lambda_{\max }^{-1}\left(G B_{u}\right)^{-1}}$. It is shown from (28) that there exists an instant $t_{f}=2 \gamma^{-1} \sqrt{V(0)}$ such that $V(t)=0$ when $t \geq$ $t_{f}$. Thus, this indicates that the system trajectories can be driven onto the predefined sliding surface in finite time, thereby completing the proof.

Remark 9. For the choice of $\gamma$, there is theoretically no limitation provided that $0<\gamma<\infty$, knowing that the larger $\gamma$ 
is the higher the control amplitude is. However, by a practical point of view, the choice of $\gamma$ is related to the physical limits of the control and the dynamics of the system. For the detailed description, we can refer to [25], in which the problem of transient time is studied in higher order sliding modes context.

\section{Numerical Examples}

In this section, we present two numerical examples to illustrate the effectiveness of previous derived results.

Example 1. Consider the following Lur'e singularly perturbed system (1)-(2) given by

$$
\begin{aligned}
& E_{\varepsilon}=\left(\begin{array}{ll}
1 & 0 \\
0 & \varepsilon
\end{array}\right), \\
& A=\left(\begin{array}{ll}
1 & -1 \\
3 & -1
\end{array}\right) \text {, } \\
& B=\left(\begin{array}{ll}
1 & 0 \\
0 & 1
\end{array}\right) \text {, } \\
& B_{u}=\left(\begin{array}{l}
1 \\
2
\end{array}\right) \text {, } \\
& C=\left(\begin{array}{ll}
1 & 0 \\
0 & 1
\end{array}\right), \\
& \varphi=\left(\frac{x_{1}\left|x_{1}\right|}{1+4 x_{1}^{2}} \frac{\left|x_{2}\right| x_{2}}{1+4 x_{2}^{2}}\right)^{T} .
\end{aligned}
$$

After some mathematical manipulations, it is easy to see that $\varphi$ satisfies the sector condition (4) with

$$
L=\left(\begin{array}{cc}
0.25 & 0 \\
0 & 0.25
\end{array}\right)
$$

For simplicity, set $G=B_{u}^{T} X$ with $X=I_{2}$. By using the proposed sliding mode control algorithm, we solve the matrix inequality (18) and obtain some parameters as follows:

$$
\begin{aligned}
& X=\left(\begin{array}{cc}
11.5779 & 0 \\
-11.4501 & 6.2760
\end{array}\right), \\
& \Gamma=\left(\begin{array}{cc}
20.1539 & 0 \\
0 & 23.7998
\end{array}\right), \\
& \Lambda=\left(\begin{array}{cc}
-4.8178 & 0 \\
0 & 2.7009
\end{array}\right), \\
& \eta=41.5971 .
\end{aligned}
$$

The state feedback control gain matrix is given by

$$
K=\left(\begin{array}{ll}
-0.2780 & 0.1253
\end{array}\right) \text {. }
$$

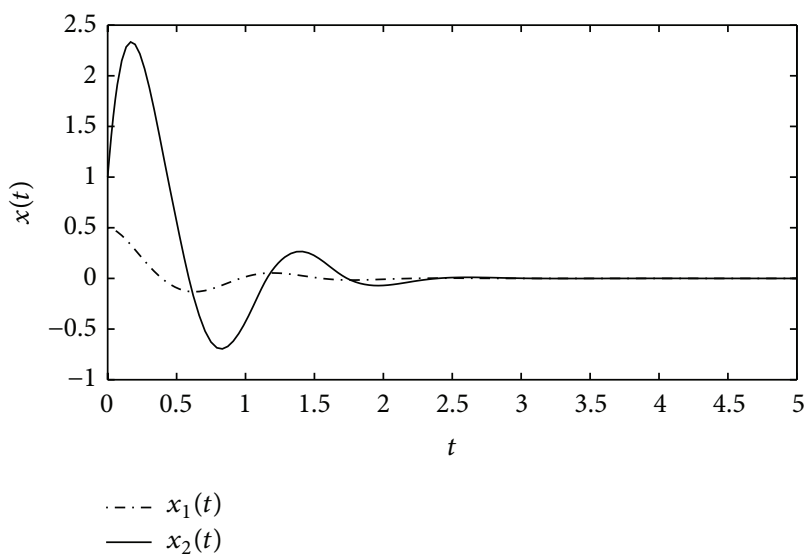

FIgURE 1: States of the closed-loop system.

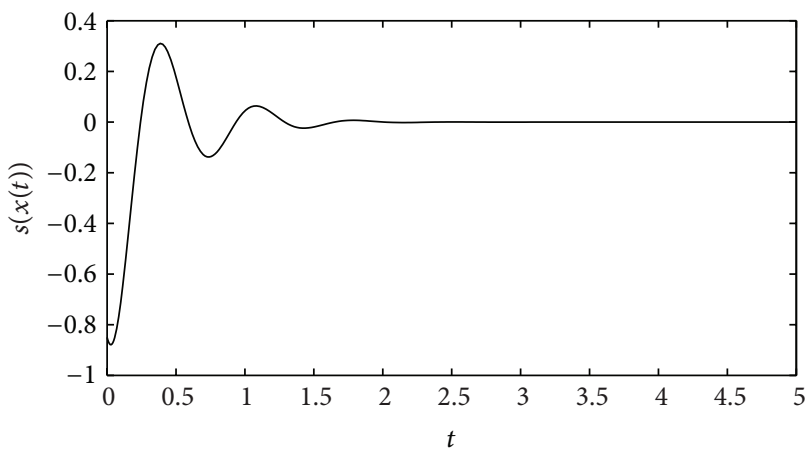

FIGURE 2: State of the sliding mode variable.

Using the bisectional search algorithm in [9], an upper bound $\varepsilon^{*}=0.2597$ can be obtained. This indicates that sliding mode dynamics is absolutely stable for $0<\varepsilon<\varepsilon^{*}=0.2597$. By Further computation, the sliding manifold can be obtained as

$$
\begin{aligned}
s(x(t))= & \left(\begin{array}{ll}
1 & 0.5192
\end{array}\right) x(t) \\
& -\int_{0}^{t}\left(\begin{array}{ll}
-0.3900 & 1.1457
\end{array}\right) x(\tau) d \tau .
\end{aligned}
$$

In case of $\gamma=0.2$, given the initial condition $x(0)=\left(\begin{array}{ll}0.5 & 1\end{array}\right)^{T}$, then the simulations for the closed-loop system and sliding mode surface function are shown in Figures 1-2, respectively. As shown in the simulations, the closed loop is asymptotically stable.

Example 2. Consider the following inverted pendulum controlled by a DC-Motor plant via a gear train in [29]:

$$
\begin{aligned}
\frac{d^{2} \theta_{p}(t)}{d t^{2}} & =-\frac{g}{l} \sin \theta_{p}(t)+\frac{N K_{m}}{m l^{2}} I_{a}(t), \\
L_{a} \frac{d I_{a}(t)}{d t} & =-K_{b} N \frac{d \theta_{p}(t)}{d t}-R_{a} I_{a}(t)+u(t),
\end{aligned}
$$

where $g=9.8 \mathrm{~m} / \mathrm{s}^{2}, l=1 \mathrm{~m}, m=1 \mathrm{~kg}, N=10, K_{m}=$ $0.1 \mathrm{Nm} / \mathrm{A}, K_{b}=0.1 \mathrm{Vs} / \mathrm{rad}$, and $R_{a}=1 \Omega, L_{a}=\varepsilon m H$. Let 
$x_{1}=\theta_{p}$, let $x_{2}=\dot{\theta}_{p}$, and let $x_{3}=\dot{I}_{a}$. Then, we have the state space form with

$$
\begin{aligned}
A & =\left(\begin{array}{ccc}
0 & 1 & 0 \\
-9.8 & 0 & 1 \\
0 & -1 & -1
\end{array}\right), \\
B & =\left(\begin{array}{lll}
0 & 9.8 & 0
\end{array}\right)^{T} \\
B_{u} & =\left(\begin{array}{lll}
0 & 0 & 1
\end{array}\right)^{T} \\
C & =\left(\begin{array}{lll}
-1 & 0 & 0
\end{array}\right),
\end{aligned}
$$

and $\varphi=\sin \left(x_{1}\right)+x_{1}$ satisfying the sector condition (4) with $L=2$. Set $G=B_{u}^{T} X$ with $X=I_{3}$. By using the proposed sliding mode control algorithm, we solve the matrix inequality (18) and obtain some parameters as follows:

$$
\begin{aligned}
X & =\left(\begin{array}{ccc}
5.3168 & 0.4502 & 0 \\
0.4502 & 0.5325 & 0 \\
0.1448 & 3.5995 & 3.4233
\end{array}\right), \\
\Gamma & =2.8656 \\
\Lambda & =5.1071 \\
\eta & =12.6685
\end{aligned}
$$

The state feedback control gain matrix is given by

$$
K=\left(\begin{array}{lll}
-2.9351 & 0.1824 & 0.3178
\end{array}\right) \text {. }
$$

Using the bisectional search algorithm in [9], an upper bound $\varepsilon^{*}=0.3517$ can be obtained. This indicates that sliding mode dynamics is absolutely stable for $0<\varepsilon<\varepsilon^{*}=0.3517$. By Further computation, the sliding manifold can be obtained as

$$
\begin{aligned}
s(x(t))= & \left(\begin{array}{lll}
0 & 0 & 0.3517
\end{array}\right) x(t) \\
& +\int_{0}^{t}\left(\begin{array}{llll}
0.2317 & 0.9856 & 0.9749
\end{array}\right) x(\tau) d \tau .
\end{aligned}
$$

In case of $\eta=0.2$, given the initial condition $x(0)=$ $\left(\begin{array}{lll}-1.5 & 0.8 & 1\end{array}\right)^{T}$, then the simulation for the states of the closed-loop system is shown in Figure 3, which shows that the controlled system can converge to zero under the designed sliding mode control law. Figure 4 gives the trajectory of the sliding mode surface function.

From these numerical studies, it is clear that the presented integral sliding mode control method eliminated the effects of sector-constrained conditions and guaranteed the asymptotic stability of the closed-loop systems. In addition, there is no equality constraint involved in matrix inequality; thus numerical problem when computing matrix inequality can be avoided. The effectiveness of the proposed method is shown clearly.

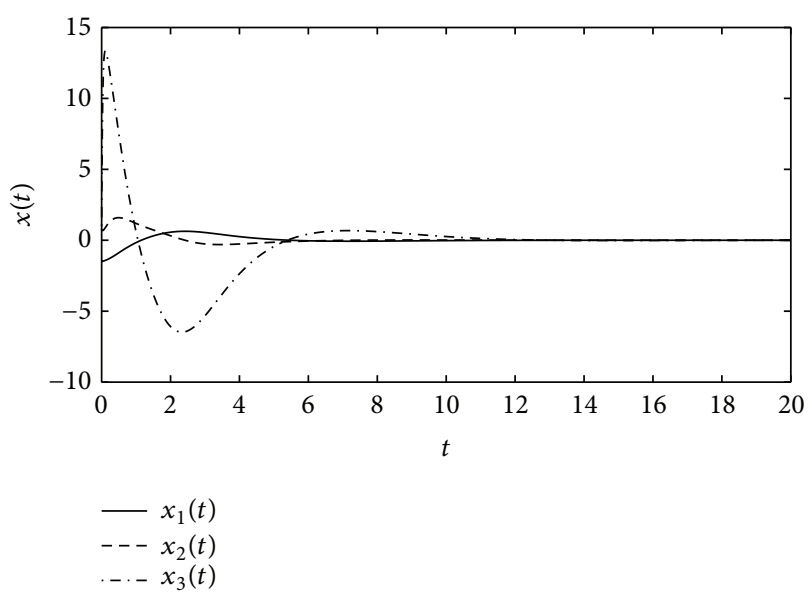

FIGURE 3: States of the closed-loop system.

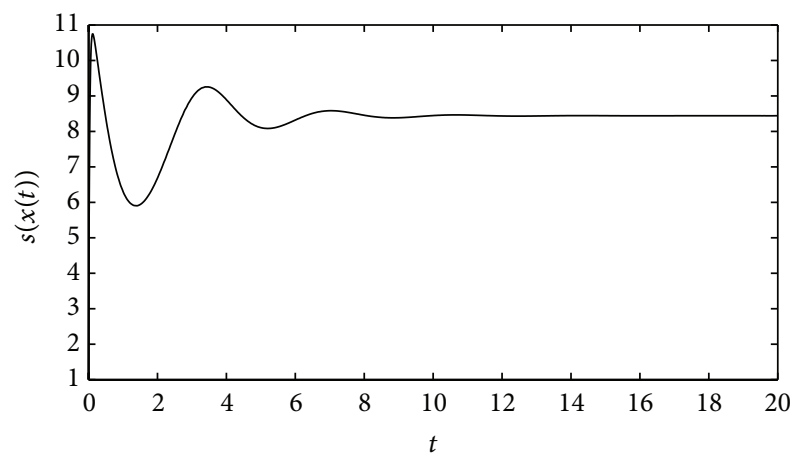

FIGURE 4: State of the sliding mode variable.

\section{Conclusion}

This paper has considered integral sliding mode control for Lur'e singularly perturbed systems with sector-constrained condition. We have constructed a proper integral sliding manifold and proposed a stability criterion expressed in terms of $\varepsilon$-independent matrix inequalities. A sliding mode control law has been synthesized such that the trajectory of the resulting closed-loop system is driven toward the manifold in finite time. The given examples have illustrated the effectiveness and advantages of the obtained method.

\section{Conflict of Interests}

The authors declare that there is no conflict of interests regarding the publication of this paper.

\section{Acknowledgments}

This paper is supported by the National Science Foundation of China (11171113), the Soft Science Research Program of Henan Province (142400411358), and the Natural Science Foundation of Henan Province (142300410324 and 142300410464). 


\section{References}

[1] P. V. Kokotovic, H. K. Khalil, and J. O’Reilly, Singular Perturbation Methods in Control: Analysis and Design, Academic Press, London, UK, 1986.

[2] D. S. Naidu, "Singular perturbations and time scales in control theory and applications: an overview," Dynamics of Continuous, Discrete and Impulsive Systems Series B: Application \& Algorithm, vol. 9, no. 2, pp. 233-278, 2002.

[3] P. Shi, S.-P. Shue, and R. K. Agarwal, "Robust disturbance attenuation with stability for a class of uncertain singularly perturbed systems," International Journal of Control, vol. 70, no. 6, pp. 873891, 1998.

[4] Z. H. Shao, "Robust stability of two-time-scale systems with nonlinear uncertainties," IEEE Transactions on Automatic Control, vol. 49, no. 2, pp. 258-261, 2004.

[5] Z. H. Shao and M. E. Sawan, "Stabilisation of uncertain singularly perturbed systems," IEE Proceedings: Control Theory and Applications, vol. 153, no. 1, pp. 99-103, 2006.

[6] Y. H. Chen and J. S. Chen, "Robust composite control for singularly perturbed systems with time-varying uncertainties," Journal of Dynamic Systems, Measurement, and Control, vol. 117, no. 4, pp. 445-452, 1995.

[7] S. J. Chen and J. L. Lin, "Maximal stability bounds of singularly perturbed systems," Journal of the Franklin InstituteEngineering and Applied Mathematics, vol. 336, no. 8, pp. 12091218, 1999.

[8] Z. M. Wang, W. Liu, H. H. Dai, and D. S. Naidu, "Robust stabilization of model-based uncertain singularly perturbed systems with networked time-delay," in Proceedings of the 48th IEEE Conference on Decision and Control Conference, pp. 7917-7922, Shanghai, China, December 2009.

[9] C. C. Yang, Q. L. Zhang, J. Sun, and T. Y. Chai, "Lur'e lyapunov function and absolute stability criterion for Lur'e singularly perturbed systems," Institute of Electrical and Electronics Engineers. Transactions on Automatic Control, vol. 56, no. 11, pp. 26662671, 2011.

[10] H. D. Tuan and S. Hosoe, "Multivariable circle criteria for multiparameter singularly perturbed systems," IEEE Transactions on Automatic Control, vol. 45, no. 4, pp. 720-725, 2000.

[11] V. R. Saksena and P. V. Kokotovic, "Singular perturbation of the Popov-Kalman-Yakubovich lemma," Systems \& Control Letters, vol. 1, no. 1, pp. 65-68, 1981/82.

[12] G. Garcia, J. Daafouz, and J. Bernussou, " $H_{2}$ guaranteed cost control for singularly perturbed uncertain systems," IEEE Transactions on Automatic Control, vol. 43, no. 9, pp. 1323-1329, 1998.

[13] M. G. Dmitriev and G. A. Kurina, "Singular perturbations in control problems," Automation and Remote Control, vol. 67, no. 1, pp. 1-43, 2006.

[14] H. P. Liu, F. C. Sun, and Z. Q. Sun, "Stability analysis and synthesis of fuzzy singularly perturbed systems," IEEE Transactions on Fuzzy Systems, vol. 13, no. 2, pp. 273-284, 2005.

[15] K.-J. Lin and T.-H. S. Li, "Stabilization of uncertain singularly perturbed systems with pole-placement constraints," IEEE Transactions on Circuits and Systems II: Express Briefs, vol. 53, no. 9, pp. 916-920, 2006.

[16] H. Singh, R. H. Brown, D. S. Naidu, and J. A. Heinen, "Robust stability of singularly, perturbed state feedback systems using unified approach," IEE Proceedings: Control Theory and Applications, vol. 148, no. 5, pp. 391-396, 2001.
[17] V. I. Utkin, Sliding Modes in Control and Optimization, Communications and Control Engineering Series, Springer, Berlin, Germany, 1992.

[18] V. I. Utkin, J. Guldner, and J. Shi, Sliding Modes in Electromechanical Systems, Taylor and Francis, London, UK, 1999.

[19] L. Wu and W. X. Zheng, "Passivity-based sliding mode control of uncertain singular time-delay systems," Automatica, vol. 45, no. 9, pp. 2120-2127, 2009.

[20] Y. Niu, D. W. C. Ho, and J. Lam, "Robust integral sliding mode control for uncertain stochastic systems with time-varying delay," Automatica, vol. 41, no. 5, pp. 873-880, 2005.

[21] J. Huang, L. N. Sun, Z. Z. Han, and L. P. Liu, "Adaptive terminal sliding mode control for nonlinear differential inclusion systems with disturbance," Nonlinear Dynamics, vol. 72, no. 1-2, pp. 221-228, 2013.

[22] B. Bandyopadhyay, F. Deepak, and Y. J. Park, "A robust algorithm against actuator saturation using integral sliding mode and composite nonlinear feedback," in Proceedings of the 17th World Congress the International Federation of Automatic Control, pp. 14174-14179, Seoul, Republic of Korea, July 2008.

[23] Y. Gao, B. Sun, and G. Lu, "Passivity-based integral slidingmode control of uncertain singularly perturbed systems," IEEE Transactions on Circuits and Systems II: Express Briefs, vol. 58, no. 6, pp. 386-390, 2011.

[24] F. Castaños and L. Fridman, "Analysis and design of integral sliding manifolds for systems with unmatched perturbations," Institute of Electrical and Electronics Engineers. Transactions on Automatic Control, vol. 51, no. 5, pp. 853-858, 2006.

[25] A. Levant, "Transient adjustment of high-order sliding modes," in Proceedings of the International Workshop on Variable Structure Systems (VSS '04), Vilanova i la Geltru, Spain, 2004.

[26] C. Yang, Q. Zhang, Y. Lin, and L. Zhou, "Positive realness and absolute stability problem of descriptor systems," IEEE Transactions on Circuits and Systems. I. Regular Papers, vol. 54, no. 5, pp. 1142-1149, 2007.

[27] C. Yang, Q. Zhang, and L. Zhou, "Strongly absolute stability of Lur'e descriptor systems: popov-type criteria," International Journal of Robust and Nonlinear Control, vol. 19, no. 7, pp. 786806, 2009.

[28] L. Xie, M. Fu, and C. E. de Souza, " $H_{\infty}$ control and quadratic stabilization of systems with parameter uncertainty via output feedback," IEEE Transactions on Automatic Control, vol. 37, no. 8, pp. 1253-1256, 1992.

[29] S. H. Zak and C. A. MacCarley, "State-feedback control of nonlinear systems," International Journal of Control, vol. 43, no. 5, pp. 1497-1514, 1986. 


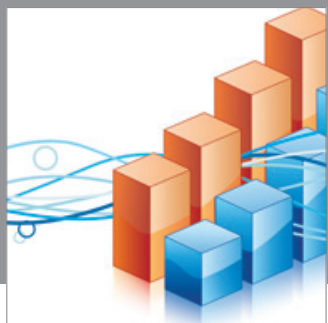

Advances in

Operations Research

mansans

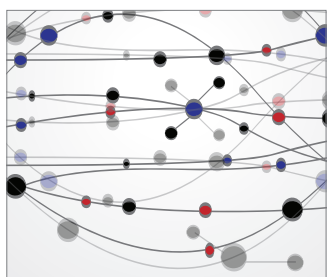

The Scientific World Journal
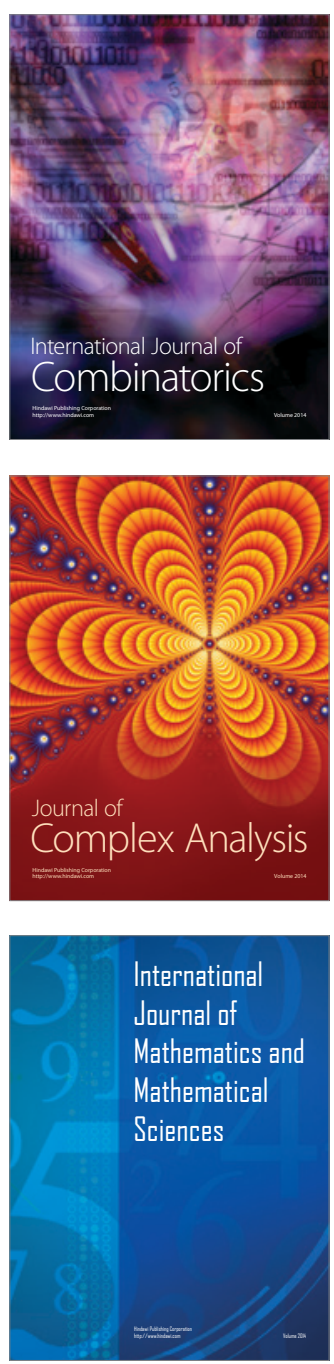
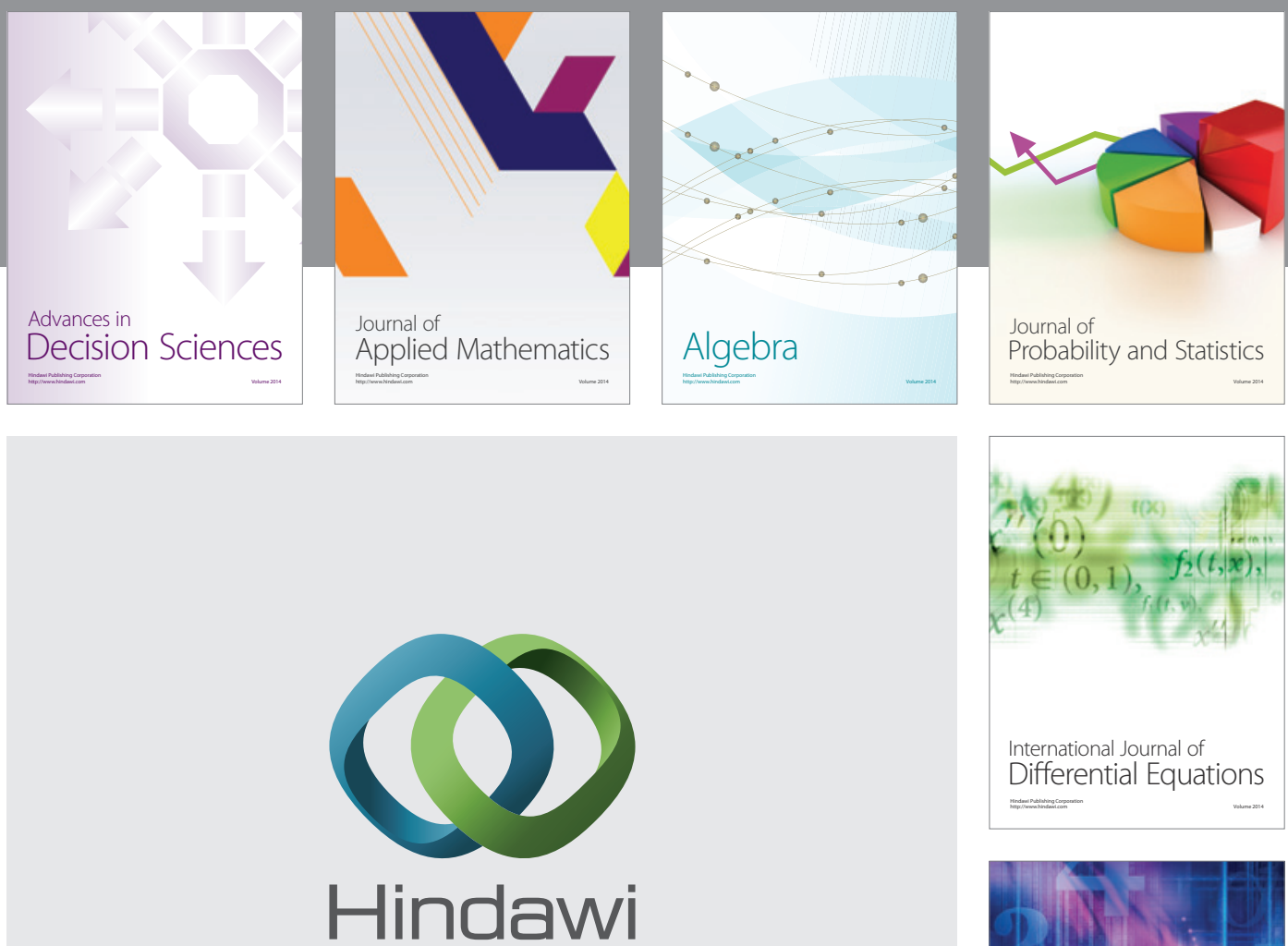

Submit your manuscripts at http://www.hindawi.com
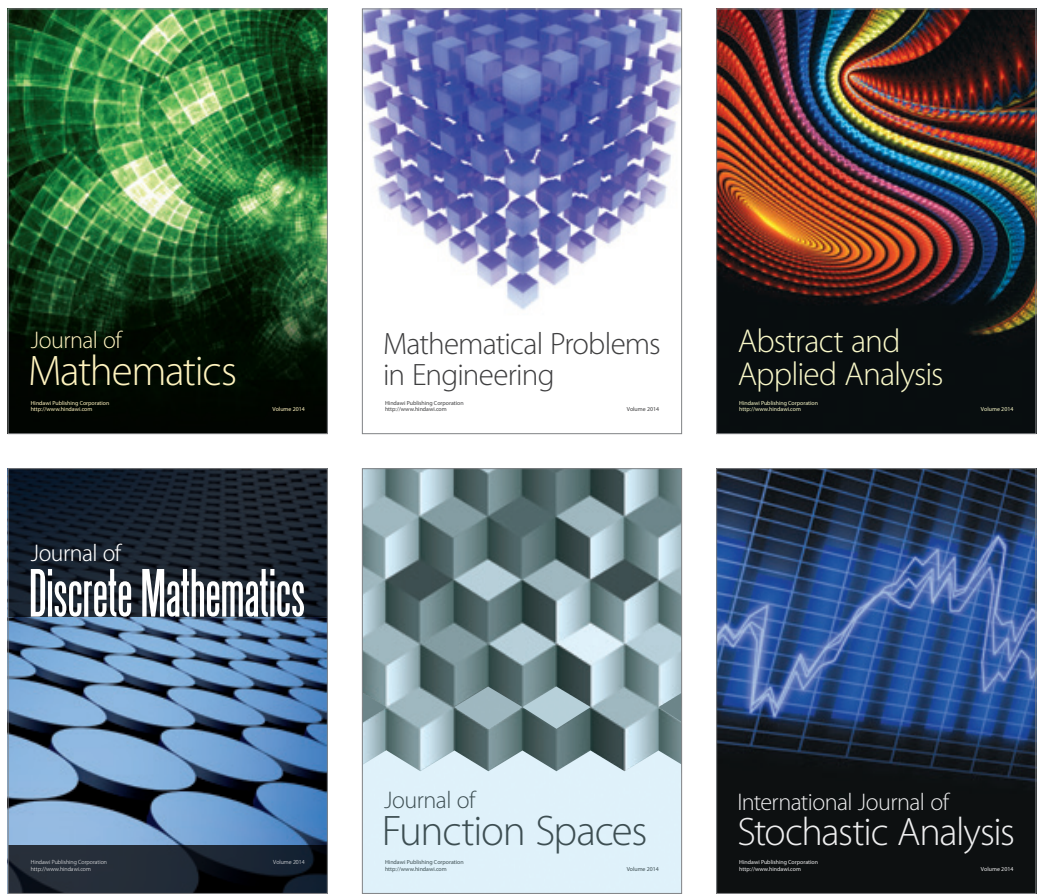

Journal of

Function Spaces

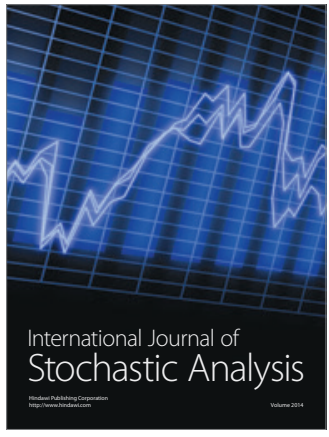

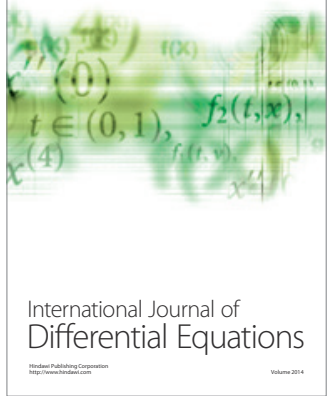
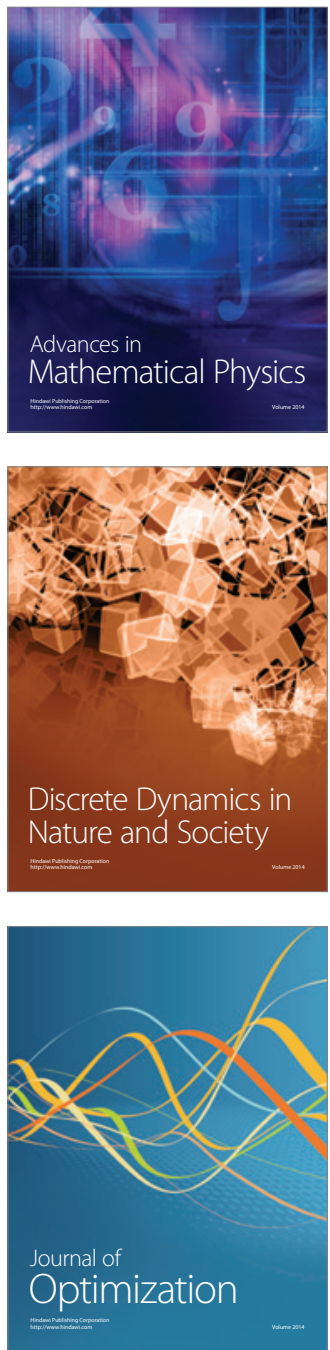